\title{
Grußworte zum Medientag 2019
}

\author{
Vizerektor Univ.-Prof. Dr. Bernhard Fügenschuh
}

Sehr geehrte Damen und Herren,

die Innsbruck Media Studies sind ein ausgezeichnetes und lange bewährtes Lehrangebot der Universität Innsbruck, das jeweils durch die Medientage noch zusätzlich geadelt wird. Dies zeigt sich nicht zuletzt an den namhaften Vertreterinnen und Vertretern aus der Medienlandschaft: Alexandra Föderl-Schmid, Bernhard Heinzlmaier, Hermann Petz, Clemens Pig und Martina Marx als DiskutantInnen am Diskussionspodium, moderiert von Georg Laich.

Das Thema in diesem Jahr „YouTube, Twitch und Netflix: Jugendliche Mediennutzung als Herausforderung für den Qualitätsjournalismus“. Drei beispielhafte Internetformate, eine konkret angesprochene Berufsgruppe. Journalismus ist gefordert als Übersetzer immer komplexerer Inhalte in die breite Gesellschaft. Qualitätsjournalismus, der sich an ausreichend ermittelten Fakten orientiert, die, mit den nötigen Mitteln ausgestattet, akribisch recherchiert wurden. Hier schließt sich der Kreis wieder mit der Gesellschaft, der diese Qualität wichtig und daher auch etwas wert sein muss. Es muss der Anspruch sein, dass das Wort Journalismus per se Ausdruck für Qualität ist und sich der Zusatz so schnell wie möglich erübrigt.

Doch Qualität kann nicht nur bei spezifischen Berufsgruppen oder exklusiven Personengruppen aufgehängt sein. Durchaus interessant kann im Titel der Veranstaltung die Verknüpfung von jugendlichem Medienkonsum und Journalismus betrachtet werden. Sind es die gleichen qualitätshinterlegten Medien, wenn wir von diesbezüglichem jugendlichen Konsum sprechen? Oder sind es kaum qualitätskontrollierbare aber umso konsumierbarere Medienangebote? Auch hier muss sich unser Konsumverhalten ändern und über frühe Bildungs- und Ausbildungsangebote dieses zu schärfen und den vielfältigen Angeboten eine kritisch-reflektierte Betrachtung entgegenstellen. Für uns

Katharina Gallner-Holzmann, Theo Hug, Günther Pallaver (Hg.):

Jugendliche Mediennutzung und die Zukunft des Qualitätsjournalismus

(C) 2020 innsbruck university press, ISBN 978-3-99106-014-7, DOI 10.15203/99106-014-7 
als Universität muss es nicht zuletzt ein zentrales Anliegen sein, diejenigen Personen auszubilden, die dieses Verständnis über Medienkonsum in alle Bildungseinrichtungen tragen um den Wert von Journalismus - in neuen und alten Medien - als zentrale Komponente einer funktionierenden Demokratie nachhaltig zu stärken.

Abschließend möchte ich Theo Hug und seinem Team herzlich für die Organisation und das stete Engagement danken und wünsche kritischen Austausch zu einem kritischen Thema. 